\title{
Laser-driven shock experiments to investigate mitigation ability of polymeric foams
}

\author{
Pierre Pradel $^{1, *}$, Frédéric Malaise ${ }^{1}$, and Thibaut de Rességuier ${ }^{2}$ \\ ${ }^{1}$ CEA CESTA, 15 avenue des Sablières CS60001, 33116 Le Barp Cedex, France \\ ${ }^{2}$ Institut P' UPR3346 CNRS-Université de Poitiers-ENSMA, 11 boulevard Marie et Pierre Curie, 86962 Futuroscope Chasseneuil \\ Cedex, France
}

\begin{abstract}
Polymeric foams are widely used in many industrial fields as thermal insulators, structural materials or shock wave mitigators. Polymeric foams would be valuable candidates to protect structures against intense mechanical stress wave loadings generated by laser irradiation or high velocity impact of very small debris. This article presents the results of laser-driven shock experiments performed on polymeric foams to investigate their mitigation ability. The targets consisted of thin aluminum front plate ( $250 \mu \mathrm{m}$-thickness), $1 \mathrm{~mm}$ and $2 \mathrm{~mm}$-thick samples made of expanded polyurethane foam $\left(320 \mathrm{~kg} / \mathrm{m}^{3}\right)$ or syntactic epoxy foam $\left(624 \mathrm{~kg} / \mathrm{m}^{3}\right)$, and $12 \mu \mathrm{m}$-thick aluminum foil. The laser beam provided $20 \mathrm{~J}$ in $25 \mathrm{~ns}$ and was shot through water confinement of the front plate. The dynamic responses of the foams were investigated by measuring time-velocity profiles at the rear surface. Preliminary tests were performed on thin aluminum plate in order to calibrate the stress wave loadings. A dynamic explicit one-dimensional hydrocode was used to simulate the experiments and validate the calibration of pressure generated under laser irradiation. Then, the numerical simulations were used to analyze the velocity profiles recorded at the rear surface of both foams. The dynamic macroscopic response of the foams was described by a phenomenological compaction model. The model has been validated by numerical correlations with the experimental results. The input pressure (front aluminum plate) and the output one (fictitious PMMA plate placed behind foam samples) were compared by help of numerical simulations. The ratio between input and output pressures could achieve 75. Polyurethane foam better mitigated shock waves below $2 \mathrm{GPa}$, and epoxy foam was better above $2 \mathrm{GPa}$.
\end{abstract}

\section{Introduction}

Polymeric foams are commonly used in aerospace, automotive [1, 2], marine [3] and military industries [4] for the protection against crashes or high velocity impacts. Their light weight and their energy absorption abilities are of great interest to develop efficient systems that mitigate the effects of intense (several GPa) and brief (1-1000 ns) stress wave loadings. At CEA CESTA, these loadings are representative of the ones that will be carried out with the Laser MégaJoule (LMJ). Effective protection systems against laser irradiation or very high velocity impacts of small debris must be developed to protect some diagnostics or samples support devices.

The expected devices consist of a metal shield, to interact with the laser beam or debris, and a mitigator layer, to reduce the intensity of the stress waves transmitted to the component to protect. A rigid closed cell polyurethane foam $\left(320 \mathrm{~kg} / \mathrm{m}^{3}\right)$ and a syntactic epoxy foam $\left(624 \mathrm{~kg} / \mathrm{m}^{3}\right)$ have been chosen for their energy absorption abilities.

Previous works permit to identify the deformation mechanisms of these foams under quasi-static loadings [3, 5, 6] or Split Hopkinson Pressure Bar experiments [3, 7]. Several gas gun experiments have been performed to determine shock Hugoniot $[8,9]$. Although these experiments

\footnotetext{
*e-mail: pierre.pradel@cea.fr
}

are accurate to study the behaviour of the foams at high strain rates, the load durations are not completely representative of laser irradiation or high velocity impacts.

The objective of this paper is to investigate the dynamic behaviour and the mitigation ability of both polymeric foams by performing laser-driven shock experiments. Numerical simulations have been carried out by using a phenomenological compaction model. Accordance between experimental and numerical data is presented. Input and output pressures have then been calculated and compared to investigate the mitigation ability of the two foams.

\section{Experimental}

\subsection{Materials}

The mitigation properties of two polymeric foams have been investigated during this study. The polyurethane foam is a closed cell foam obtained by mixing water with an isocyanate (MDI) and an alcohol. The reaction between water and isocyanate produces bubbles of carbon dioxide, which constitute the structure of the foam. The cells are spherical and their diameter is between a few tens and a few hundred microns (see 2D tomography in Fig. 1). The density is about $320 \pm 20 \mathrm{~kg} / \mathrm{m}^{3}$ and its porosity is around 
$66 \%$. According to literature data [10], the density of fully dense polyurethane is $1240 \pm 40 \mathrm{~kg} / \mathrm{m}^{3}$.

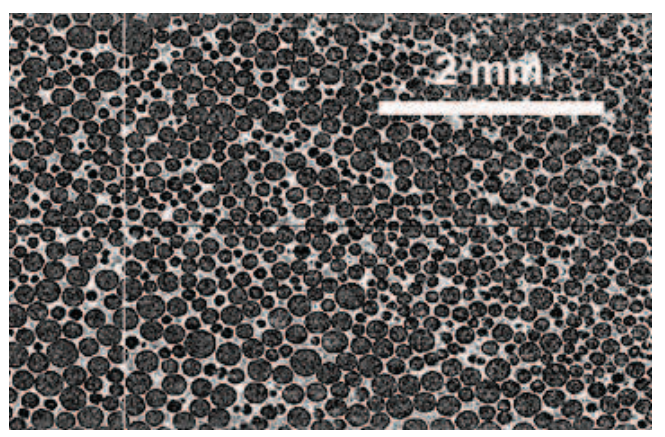

Fig. 1. Microstructure of polyurethane foam obtained by X-ray tomography.

The second foam is a syntactic epoxy foam obtained by adding glass microspheres during the polymerization reaction of an epoxy resin. The diameter of the microspheres is about $60 \mu \mathrm{m}$, and the thickness of the walls of the spheres is around $1 \mu \mathrm{m}$ (see optical microscopy in Fig. 2). The density of the foam is about $624 \pm 10 \mathrm{~kg} / \mathrm{m}^{3}$ and the porosity is around $62 \%$. The density of fully dense epoxy is between 1190 and $1490 \mathrm{~kg} / \mathrm{m}^{3}$.

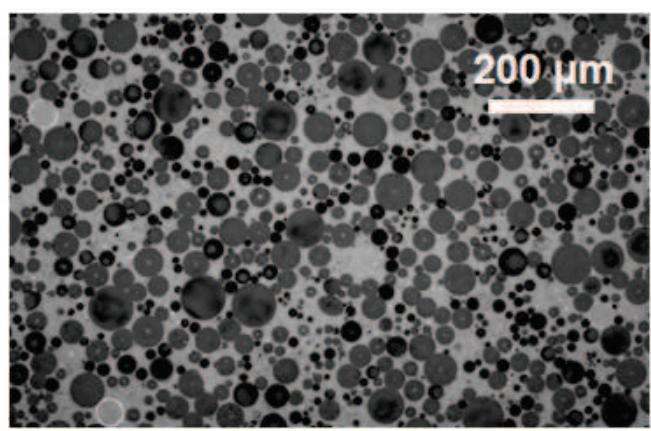

Fig. 2. Microstructure of epoxy foam obtained by optical microscopy.

\subsection{Experimental set-up}

The experimental set-up used for these laser-driven shock experiments is shown in Fig. 3. The targets consist of a $250 \mu \mathrm{m}$-thick aluminum front plate bonded to $1 \mathrm{~mm}$ or $2 \mathrm{~mm}$-thick foam samples. To delay the expansion of the plasma, water confined regime configuration was used by depositing a few drops of water on the front side of the aluminum plates. It permits to increase the ablation pressure and to double the pulse duration [11]. The thickness of this water confinement is about $1 \mathrm{~mm}$.

The dynamic responses of the foams have been investigated by measuring the velocity histories at the rear surface by using a VISAR (Velocity Interferometer System for Any Reflector). A $12 \mu \mathrm{m}$-thick aluminum foil is bonded to the rear surface of the samples to provide better reflection of the laser beam used by the interferometer. The thickness of the aluminum foil is small enough in

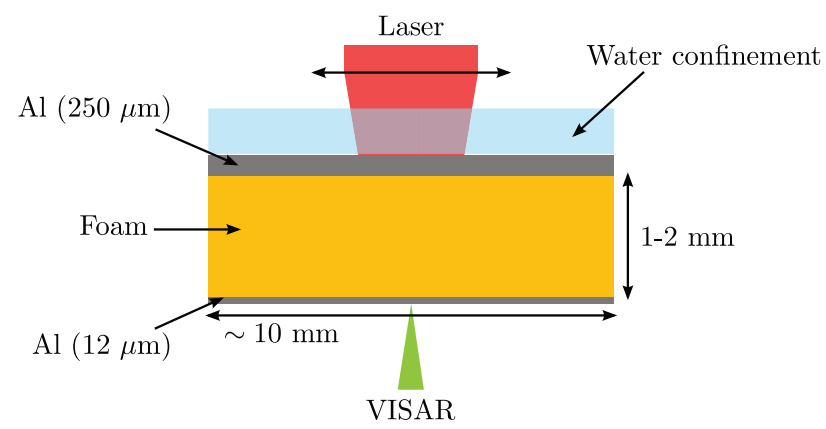

Fig. 3. Laser-driven shock experimental set-up.

front of the thickness of the foams to consider that the rear surface of the foam samples is a free surface.

Experiments have been performed with the laser of Institut P'. It delivers pulses of $20 \mathrm{~J}$ for a duration of $25 \mathrm{~ns}$ with a spot diameter of about 5-6 $\mathrm{mm}$, which corresponds to intensities of about 3-4 GW/ $\mathrm{cm}^{2}$. During the experimental campaign, the laser energy has been set between 9 and $20 \mathrm{~J}$ (Tab. 1). Taking into account the confinement, the pulse duration reaches $50 \mathrm{~ns}$ and the pressures are ranging from 0.9 to $2.6 \mathrm{GPa}$.

Table 1. Parameters of laser-driven shock experiments.

\begin{tabular}{llll}
\hline Foam & Shot number & $\begin{array}{l}\text { Thickness } \\
(\mathrm{mm})\end{array}$ & $\begin{array}{l}\text { Energy } \\
E(\mathrm{~J})\end{array}$ \\
\hline \multirow{6}{*}{ Polyurethane } & pu1000_07 & 0.971 & 16.92 \\
& pu1000_09 & 0.993 & 17.11 \\
& pu1000_10 & 0.950 & 13.16 \\
& pu2000_23 & 0.988 & 18.05 \\
& pu2000_15 & 2.018 & 18.80 \\
& pu2000_22 & 2.017 & 12.78 \\
Epoxy & epo1000_11 & 0.994 & 17.11 \\
\hline & epo1000_14 & 1.013 & 19.18 \\
& epo2000_18 & 1.990 & 18.97 \\
& epo2000_21 & 2.005 & 18.99 \\
\hline
\end{tabular}

\section{Results and discussion}

\subsection{Calibration of the stress wave loadings}

The presence of a confinement prevents the analytical calculation of the ablation pressure using the Grün formula [12]. Thus dedicated laser shots have been performed on $250 \mu \mathrm{m}$-thick aluminum samples in order to calibrate the stress wave loadings by using an inverse method. The ablation pressure, the pulse duration and the shape of the release are determined to reproduce the experimental velocity profiles, shown in Fig. 4, for two laser energies.

The calculations have been performed by using a dynamic explicit one-dimensional hydrocode. The Bushman-Lomonosov-Fortov multiphase equation of state [13] and the Steinberg-Cochran-Guinan elasticplastic constitutive model [14] represented the dynamic behaviour of aluminum. The pressure profiles are shown 


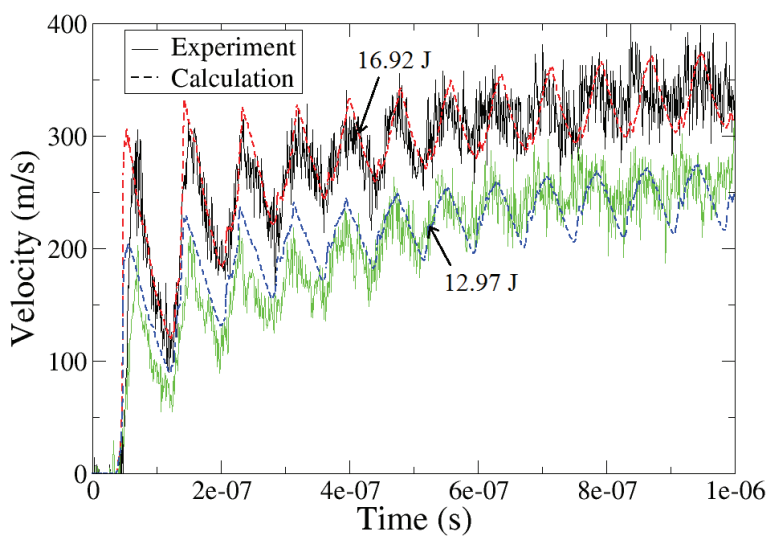

Fig. 4. Velocity profiles of aluminum free surface.

in Fig. 5. The correlations between numerical and experimental velocity profiles are fairly good (Fig. 4). It validates the ablation pressure profiles for laser energies between 13 and $17 \mathrm{~J}$.

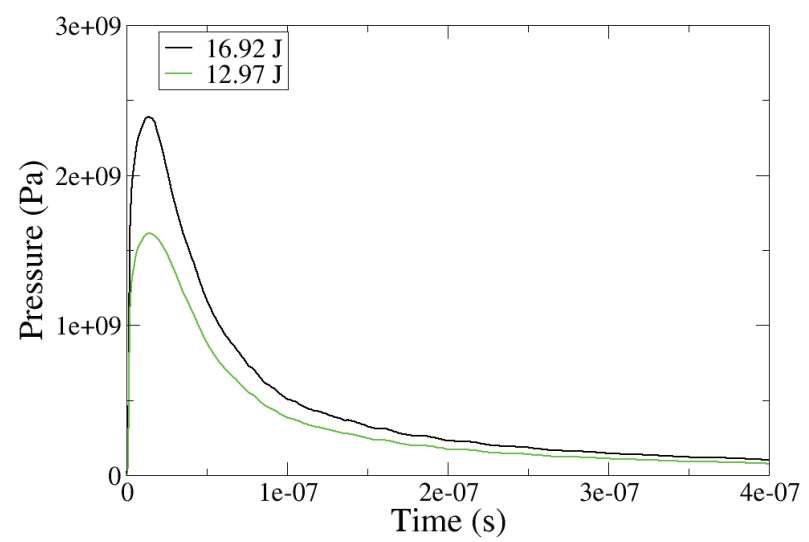

Fig. 5. Ablation pressure profiles.

\subsection{Experimental results}

Velocity profiles measured at the rear surface of $1 \mathrm{~mm}$ thick foam samples are shown in Fig. 6 and 7 for the shots \#pu1000_09 and \#epo1000_11. During the propagation through the foam, the stress wave splits in an elastic precursor and a compaction wave. The first velocity level is associated to the arrival of the elastic precursor. The level of this precursor is about $80 \mathrm{~m} / \mathrm{s}$ for polyurethane foam, and $90-100 \mathrm{~m} / \mathrm{s}$ for epoxy foam. At the rear surface of the foam, the elastic wave is reflected into release waves which interact with the compaction wave. The compaction wave is not registered during these experiments.

\subsection{Numerical results}

The dynamic behaviour of polyurethane and epoxy foams is represented by the SRI phenomenological compaction model [15]. The parameters of the models have been validated by comparisons between numerical and experimental results. The results of quasi-static, magnetic pressure

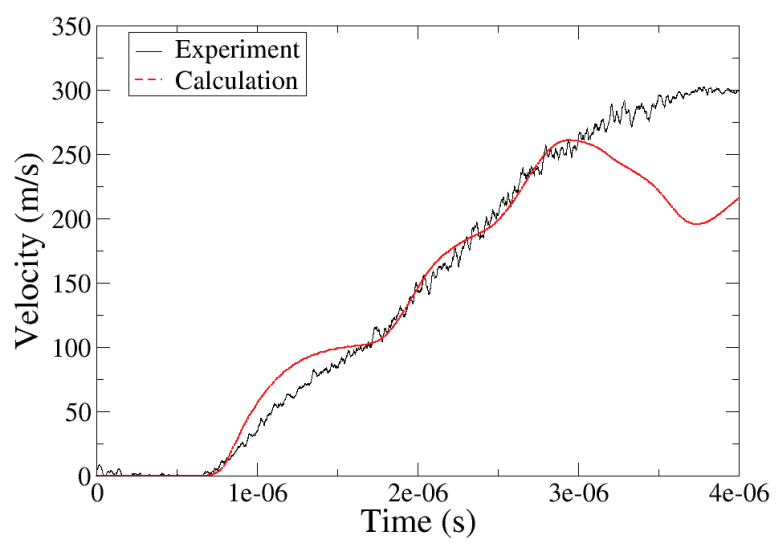

Fig. 6. Comparison between experimental and numerical velocity histories at the rear surface of polyurethane foam.

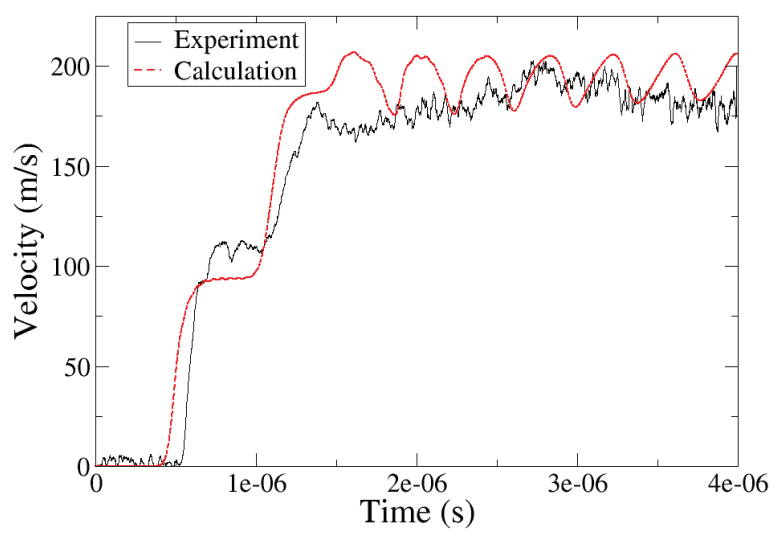

Fig. 7. Comparison between experimental and numerical velocity histories at the rear surface of epoxy foam.

and plate impact experiments have been used for this calibration. During these previous studies, the dynamic compaction threshold has been determined: it was $21 \mathrm{MPa}$ for polyurethane foam, and $72 \mathrm{MPa}$ for epoxy foam.

The laser-driven shock experiments have been simulated by using the one-dimensional hydrocode. The targets have been discretized to correctly capture the laser shock (mesh size between 1 and $40 \mu \mathrm{m}$ ). The computations have been initiated by applying the ablation pressure profiles deduced from calibration experiments. The comparisons between experimental and numerical data are shown in Fig. 6 and 7. The arrival times of the waves and the velocity levels are fairly reproduced, which validates the compaction models under laser-driven shock loadings.

\subsection{Energy absorption}

To highlight the energy absorption abilities of the foams, we have simulated the propagation of a laser shock wave through a target made of $250 \mu \mathrm{m}$-thick aluminum plate, $1 \mathrm{~mm}$-thick foam sample and $5 \mathrm{~mm}$-thick PMMA window. The results obtained for three laser energies $(9.4 \mathrm{~J}, 13.0 \mathrm{~J}$ and $16.9 \mathrm{~J}$ ) are represented in Fig. 8 . The transmitted stress wave calculated into the PMMA window depends on the 
incident stress wave and the foam properties (polyurethane or epoxy foam).

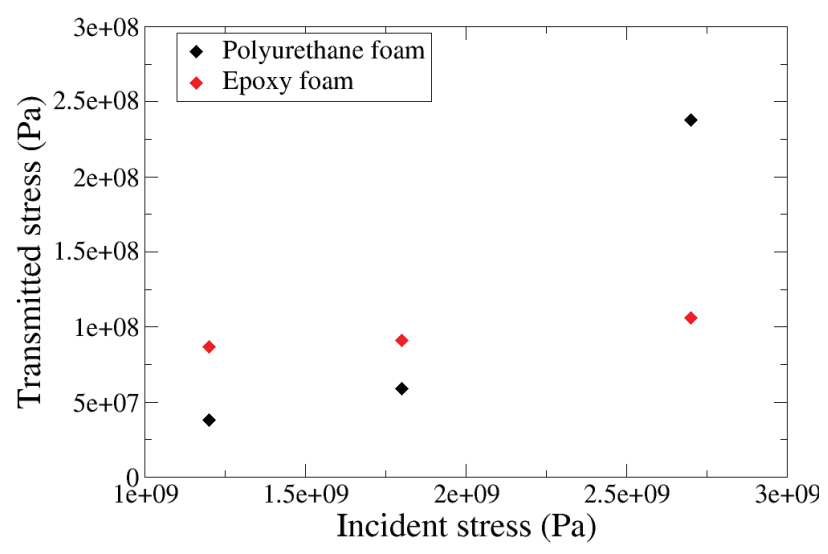

Fig. 8. Evolution of transmitted stress into the window as a function of incident stress into aluminum.

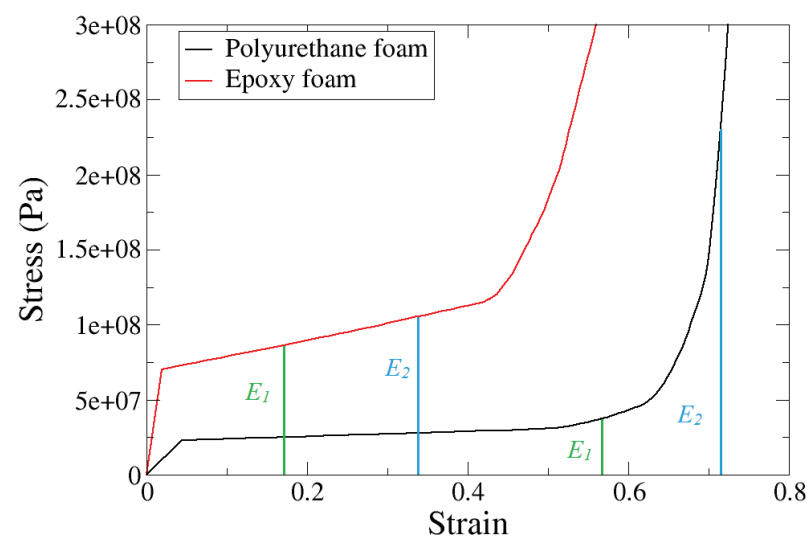

Fig. 9. Comparison of the stress-strain curves of the foams.

If the incident stresses are below $2 \mathrm{GPa}$, the transmitted stresses calculated into the PMMA window are lower with polyurethane foam. For these loadings, polyurethane foam is a better shock wave mitigator than epoxy foam. Beyond $2 \mathrm{GPa}$, it is the opposite. We notice that the mitigation is very efficient since the intensity of the stresses can be divided by a factor up to 30 using polyurethane foam, and a factor up to 25 using epoxy foam. The energy per unit volume absorbed by the foam is represented by the area under the stress-strain curve [16]. As shown in Fig. 9, since the compaction plateau of polyurethane foam is below the epoxy foam one, the transmitted peak stress is lower. This is the case when the absorbed energy is equal to $E_{1}$ (see Fig. 9). When fully densified state is achieved into polyurethane foam, epoxy foam becomes more efficient. This is the case when the absorbed energy is equal to $E_{2}$. The transmitted stress obviously depends on the foam thickness: it decreases when the foam thickness increases, and the ratio between the transmitted and the incident stresses can reach 75 if the foams thickness is $2 \mathrm{~mm}$.

\section{Conclusion}

Laser-driven shock experiments have been performed to investigate the mitigation abilities of two polymeric foams. After a calibration step on aluminum targets, the velocity profiles measured at the rear surface of both foams have been simulated. The good agreement between experimental and numerical results validated the use of the models under laser stress wave loadings. Thus, these models have been used to analyze the energy absorption abilities of the foams. Polyurethane foam is a better mitigator than epoxy foam for stress levels below $2 \mathrm{GPa}$. Above $2 \mathrm{GPa}$, it is the opposite.

\section{References}

1. F.A.O. Fernandes, R.T. Jardin, A.B. Pereira, and R.J. Alves de Sousa. Comparing the mechanical performance of synthetic and natural cellular materials. Materials \& Design, 82 335-341 (2015)

2. L. Di Landro, G. Sala, and D. Olivieri. Deformation mechanisms and energy absorption of polystyrene foams for protective helmets, Polymer Testing, 21 217-228 (2002)

3. A. Pellegrino, V.L. Tagarielli, R. Gerlach, and N. Petrinic. The mechanical response of a syntactic polyurethane foam at low and high rates of strain, International Journal of Impact Engineering, 75 214-221 (2015)

4. J.C. Gowda. A flexible syntactic foam for shock mitigation, PhD thesis, North Carolina A\&T State University (2011)

5. H. Jmal. Identification du comportement quasi-statique et dynamique de la mousse de polyuréthane au travers de modèles à mémoire, $\mathrm{PhD}$ thesis, Université de Haute Alsace (2012)

6. Z.H. Tu, V.P.W. Shim, and C.T. Lim. Plastic deformation modes in rigid polyurethane foam under static loading, International Journal of Solids and Structures, 38 9267-9279 (2001)

7. W. Chen, F. Lu, and N. Winfree. High-strain-rate compressive behavior of a rigid polyurethane foam with various densities, Experimental Mechanics, 42 65-73 (2002)

8. E. Zaretsky, Z. Asaf, E. Ran, and F. Aizik. Impact response of high density flexible polyurethane foam, International Journal of Impact Engineering, 39 1-7 (2012)

9. D.M. Dattelbaum, J.D. Coe, C.B. Kiyanda, R.L. Gustavsen, and B.M. Patterson. Reactive, anomalous compression in shocked polyurethane foams, Journal of Applied Physics, 115174908 (2014)

10. S.P. Marsh. LASL shock Hugoniot data, University of California Press (1980)

11. L. Berthe. Processus de claquage de milieux transparents sous irradiation laser. Application au choc laser en régime de confinement par eau, $\mathrm{PhD}$ thesis, Université de Paris Sud (1998)

12. J. Grün, R. Decoste, B.H. Ripin, and J. Gardner. Characteristics of ablation plasma from planar, laser-driven targets, Applied Physics Letters, 39 545-547 (1981) 
13. A.V. Bushman, I.V. Lomonosov, and V.E. Fortov. Equations of state for metals at high energy density, Institute of Chemical Physics (1992)

14. D.J. Steinberg, S.G. Cochran, and M.W. Guinan. A constitutive model for metals applicable at high-strainrate, Journal of Applied Physics 51 (1980)
15. L. Seaman, R.E. Tokheim, and D.R. Curran. Computational representation of constitutive relations for porous materials, Technical report, Stanford Research Institute (1974)

16. L.J. Gibson and M.F. Ashby. Cellular solids: Structure and properties - Second edition, Cambridge University Press (1997) 
